\title{
Demonstration of an $\mathrm{As}_{2} \mathrm{~S}_{3}$ Grating Coupler on Thin Film $\mathrm{LiNbO}_{3}$
}

\author{
Chen Zhang, Christi K. Madsen \\ Department of Electrical and Computer Engineering, Texas A\&M University, College Station, TX, USA \\ Email: jasonoehust@tamu.edu
}

How to cite this paper: Zhang, C. and Madsen, C.K. (2018) Demonstration of an $\mathrm{As}_{2} \mathrm{~S}_{3}$ Grating Coupler on Thin Film $\mathrm{LiNbO}_{3}$. Optics and Photonics Journal, 8, 111-121. https://doi.org/10.4236/opj.2018.84011

Received: January 8, 2018

Accepted: April 27, 2018

Published: April 30, 2018

Copyright $\odot 2018$ by authors and Scientific Research Publishing Inc. This work is licensed under the Creative Commons Attribution International License (CC BY 4.0).

http://creativecommons.org/licenses/by/4.0/

\section{cc) (i) Open Access}

\begin{abstract}
We present both design and experimental results for an $\mathrm{As}_{2} \mathrm{~S}_{3}$ grating coupler on a thin film $\mathrm{LiNbO}_{3}$ substrate. A basic grating coupler structure is designed with coupling efficiency of $53 \%$ to a single mode fiber. A maximum simulated coupling efficiency of $78.8 \%$ is achieved, assuming a polymer bonding process. The basic structure was fabricated, and the coupling efficiency was measured to be at least $23.4 \%$ at $1540 \mathrm{~nm}$. Some of the loss may be attributable to non-grating sources, such as waveguide tapers and testing fiber tails. A grating cavity was then measured using the grating couplers. The cavity waveguide propagation loss was $2.0 \mathrm{~dB} / \mathrm{cm}$. For a $400 \mathrm{~nm}$ thick $\mathrm{As}_{2} \mathrm{~S}_{3}$ on $500 \mathrm{~nm}$ thin film $\mathrm{LiNbO}_{3}$ on insulator, the confinement factor in the $\mathrm{LiNbO}_{3}$ crystal is $82.3 \%$ when the $\mathrm{As}_{2} \mathrm{~S}_{3}$ waveguide width is $400 \mathrm{~nm}$, showing that $\mathrm{As}_{2} \mathrm{~S}_{3}$-on-thin film $\mathrm{LiNbO}_{3}$ is an excellent candidate for thin film electro-optic applications.
\end{abstract}

\section{Keywords}

Thin Film Lithium Niobate, Arsenic Trisulfide Waveguide, Grating Coupler, Grating Cavity

\section{Introduction}

Lithium niobate $\left(\mathrm{LiNbO}_{3}\right)$ has been widely used in high-speed modulators and phased arrays due to the excellent electro-optic properties of $\mathrm{LiNbO}_{3}$ material [1] [2]. Compared with Si platform, $\mathrm{LiNbO}_{3}$ platform features high bandwidth and good temperature stability [3] [4].

Thin film $\mathrm{LiNbO}_{3}$ furthers the advantage over traditional $\mathrm{LiNbO}_{3}$ by confining mode in vertical direction. Thin film $\mathrm{LiNbO}_{3}$ is produced through ion implantation, to create crystal damage at the desired depth of bulk $\mathrm{LiNbO}_{3}$ wafer, followed by wet etching or thermal annealing [5] [6]. The technology development of thin film $\mathrm{LiNbO}_{3}$ enables ultra-compact electro-optic (EO) devices such as 
modulators and switches [5] [7] [8].

Arsenic trisulfide $\left(\mathrm{As}_{2} \mathrm{~S}_{3}\right)$ waveguide on $\mathrm{LiNbO}_{3}$ crystal is a hybrid platform for both near-infrared (near-IR) and mid-infrared (mid-IR). With certain waveguide width and thickness values, optical mode inside the waveguide partially overlaps with $\mathrm{LiNbO}_{3}$ crystal, enabling EO applications. Since standard semiconductor fabrication techniques can be applied to $\mathrm{As}_{2} \mathrm{~S}_{3}$ patterning, different $\mathrm{As}_{2} \mathrm{~S}_{3}$ structures including sidewall Bragg grating [9] and ring resonator [10] have been reported.

Coupling light between this platform and the external light source and detector should be carefully designed, in order to avoid excessive mode mismatch loss. In order to couple light into and out of different $\mathrm{As}_{2} \mathrm{~S}_{3}$ structures, a grating coupler can be used. Reference [11] has focused on the design method of $\mathrm{As}_{2} \mathrm{~S}_{3}$ grating coupler on bulk $\mathrm{LiNbO}_{3}$ substrate. In this paper, design, fabrication and measurement of $\mathrm{As}_{2} \mathrm{~S}_{3}$ grating coupler on top of thin film $\mathrm{LiNbO}_{3}$ crystal are discussed in details.

\section{Device Design}

Figure 1 illustrates an $\mathrm{As}_{2} \mathrm{~S}_{3}$ grating coupler on thin film $\mathrm{LiNbO}_{3}$ with both top view and side view. Here, $\mathrm{w}_{\mathrm{GC}}$ is the grating coupler width. $\Lambda$ stands for the grating coupler period. $d$ is the length of the grating section within each period. $t$ is the grating coupler thickness. The grating coupler duty cycle (DC) is defined as $\mathrm{d} / \Lambda$.

Light diffraction in a grating coupler follows phase matching condition. The goal of grating coupler design is to maximize the coupling efficiency between the first order light diffraction at the coupler output and a single-mode fiber (SMF) mode. Equation (1) is the grating coupler equation that builds connection between the first order diffraction angle $\theta$ and the effective refractive index of the grating coupler $n_{\text {eff }}$ [9]. Here, $\Lambda$ is grating coupler period, $\lambda$ is wavelength of light, and $n_{c}$ is cladding refractive index.

$$
\Lambda=\lambda /\left(n_{e f f}-n_{c} \sin \theta\right) .
$$

Effective refractive index of a grating coupler is determined by the material refractive index and thickness of each layer, as well as grating coupler DC. In simulation, we use $500 \mathrm{~nm}$ as $\mathrm{LiNbO}_{3}$ thin film thickness and $2 \mu \mathrm{m}$ as $\mathrm{SiO}_{2}$ layer thickness, which is used in device fabrication. Table 1 lists the refractive index of each layer at $1550 \mathrm{~nm}$ [12]. It is worth noting that extraordinary refractive index of $\mathrm{LiNbO}_{3}$ is used, since this design is carried out for TE polarization on X-cut thin film $\mathrm{LiNbO}_{3}$ and the light propagation is along $\mathrm{Y}$-axis.

There are more than one set of parameters satisfies (1), each of which results in a first diffraction field. Fimmprop (from Photon Design Inc.) is used to simulate grating coupler output field, and Python scripting is used to calculate and maximize the overlapping integral between the grating coupler output field and the Gaussian approximation in a SMF. The coupling angle is set to $12^{\circ}$. The 

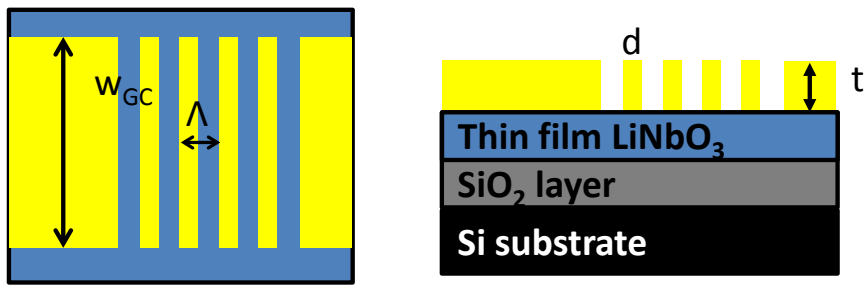

Figure 1. Top view and side view of $\mathrm{As}_{2} \mathrm{~S}_{3}$ grating coupler on thin film $\mathrm{LiNbO}_{3}$.

Table 1. Refractive index for each material in grating coupler design.

\begin{tabular}{cc}
\hline Material & Refractive index at $1550 \mathrm{~nm}$ \\
\hline $\mathrm{As}_{2} \mathrm{~S}_{3}$ & 2.4373 \\
$\mathrm{LiNbO}_{3}(\mathrm{e})$ & 2.1376 \\
$\mathrm{SiO}_{2}$ & 1.4440 \\
$\mathrm{Si}$ & 3.4777 \\
\hline
\end{tabular}

cladding index is set to 1.444 , since $\mathrm{SiO}_{2}$ is used as cladding material. The target wavelength is $1550 \mathrm{~nm}$. After optimization, grating coupler period is $850 \mathrm{~nm}$, its thickness is $400 \mathrm{~nm}$ and its DC is 0.59 . In order to further increase coupling efficiency, DC in the first several periods of the grating coupler can be tweaked (also named apodization) in order to make the grating coupler output field more resembles Gaussian approximation in a SMF [13]. After optimization, DC $=0.88$ for the first two periods; $\mathrm{DC}=0.82$ for the next two periods; $\mathrm{DC}=0.70$ for the next two periods; $\mathrm{DC}=0.59$ (unchanged) for the rest 10 periods. Compared to the uniform grating coupler, the coupling efficiency is further increased from $27.5 \%$ to $53.0 \%$. Figure 2 shows this basic $\mathrm{As}_{2} \mathrm{~S}_{3}$ grating coupler structure.

The above design methodology can be carried out targeting mid-infrared application. The optimized mid-infrared structure would have different grating period, duty cycle, and material thickness compared to above design.

Figure 3(a) shows periodicity of coupling efficiency with respect to $\mathrm{SiO}_{2}$ buffer layer thickness. The fine thickness control of this $\mathrm{SiO}_{2}$ buffer layer is critical for constructive interference between the top and bottom diffractive light. Figure $3(\mathrm{~b})$ is the spectral response of the basic grating coupler. The $3-\mathrm{dB}$ bandwidth of the basic grating coupler is $46 \mathrm{~nm}$.

Further improvements on top of the basic grating coupler structure in terms of coupling efficiency to a SMF require substantially changing the structure. One way is to introduce a Bragg reflector between substrate and grating coupler to reduce substrate leakage [13]. In our case, $\mathrm{SiO}_{2}$ can be used as low-index material and $\mathrm{As}_{2} \mathrm{~S}_{3}$ can be used as high-index material. Another way to improve the grating coupler performance is to control $\mathrm{As}_{2} \mathrm{~S}_{3}$ etching time for a specific grating depth, in order to reduce undesirable light reflection.

Since additional layers are required between $\mathrm{LiNbO}_{3}$ thin film and substrate, this improved grating coupler consists of two parts: the first part is Bragg reflector layers on a substrate; the second part is similar to the basic grating coupler 


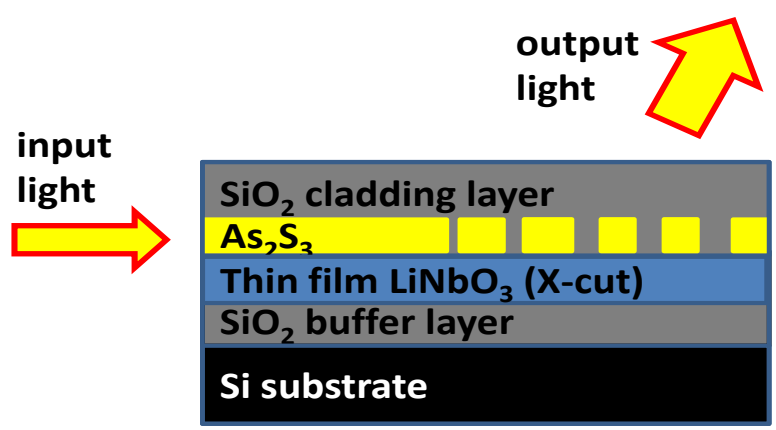

Figure 2. Basic $\mathrm{As}_{2} \mathrm{~S}_{3}$ grating coupler structure.

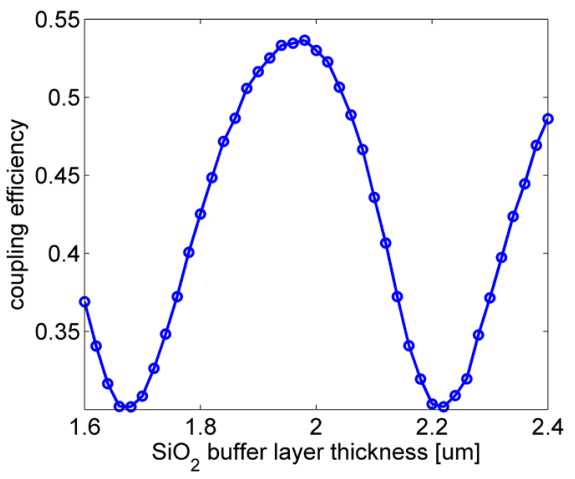

(a)

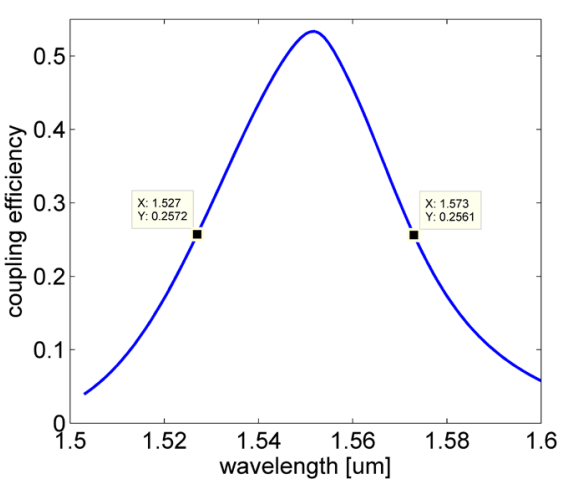

(b)

Figure 3. (a) (left) Coupling efficiency periodically changes with $\mathrm{SiO}_{2}$ buffer thickness; (b) (right) Grating coupler spectral response for the basic structure.

structure. A bonding step brings these two parts together. Figure 4 shows the improved structure implementing a Bragg reflector and controlled $\mathrm{As}_{2} \mathrm{~S}_{3}$ etching.

Benzocyclobutene (BCB) can be used as the bonding polymer. $\mathrm{BCB}$ is widely applied to low temperature wafer level bonding, with good adhesion, easy fabrication, repeatable thickness and refractive index control [14] [15]. According to Dow Chemical Company documents [16], Cyclotene (3022-46) should be used, with curing temperature $250^{\circ} \mathrm{C}$ for 60 minutes. This polymer possesses pretty good thermal stability below $350^{\circ} \mathrm{C}$. The film refractive index after curing is 1.535 at $1550 \mathrm{~nm}$.

Optical simulation of the improved grating coupler structure is carried out in Fimmprop. After optimization, the coupling efficiency is $78.8 \%$ at $1550 \mathrm{~nm}$ for TE polarization. The design parameters are listed in Table 2.

Figure 5 shows the grating coupler spectral response at coupling angle of $14^{\circ}$. The solid curve is the spectral response of the improved structure with Bragg reflector on the bottom, while the dashed curve is the spectral response of the same grating parameters without Bragg reflector. It is worth noting that the peak coupling efficiency $(80.6 \%)$ is at $1545 \mathrm{~nm}$ rather than $1550 \mathrm{~nm}$, because there are a lot of variables for the design, and each variable is discrete in the simulation with practical considerations. The apodization design makes the spectrum asymmetrical. The $3-\mathrm{dB}$ bandwidth is $60 \mathrm{~nm}$, defined by two $3-\mathrm{dB}$ drop points 


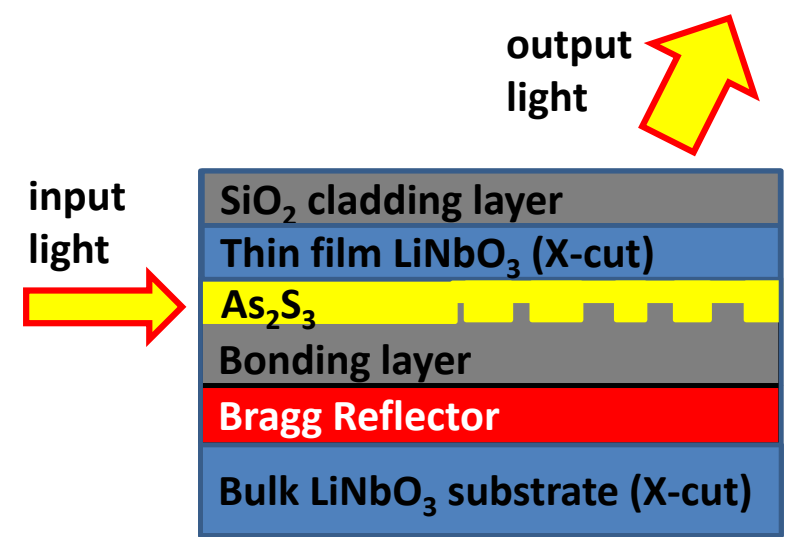

Figure 4. Improved $\mathrm{As}_{2} \mathrm{~S}_{3}$ grating coupler structure.

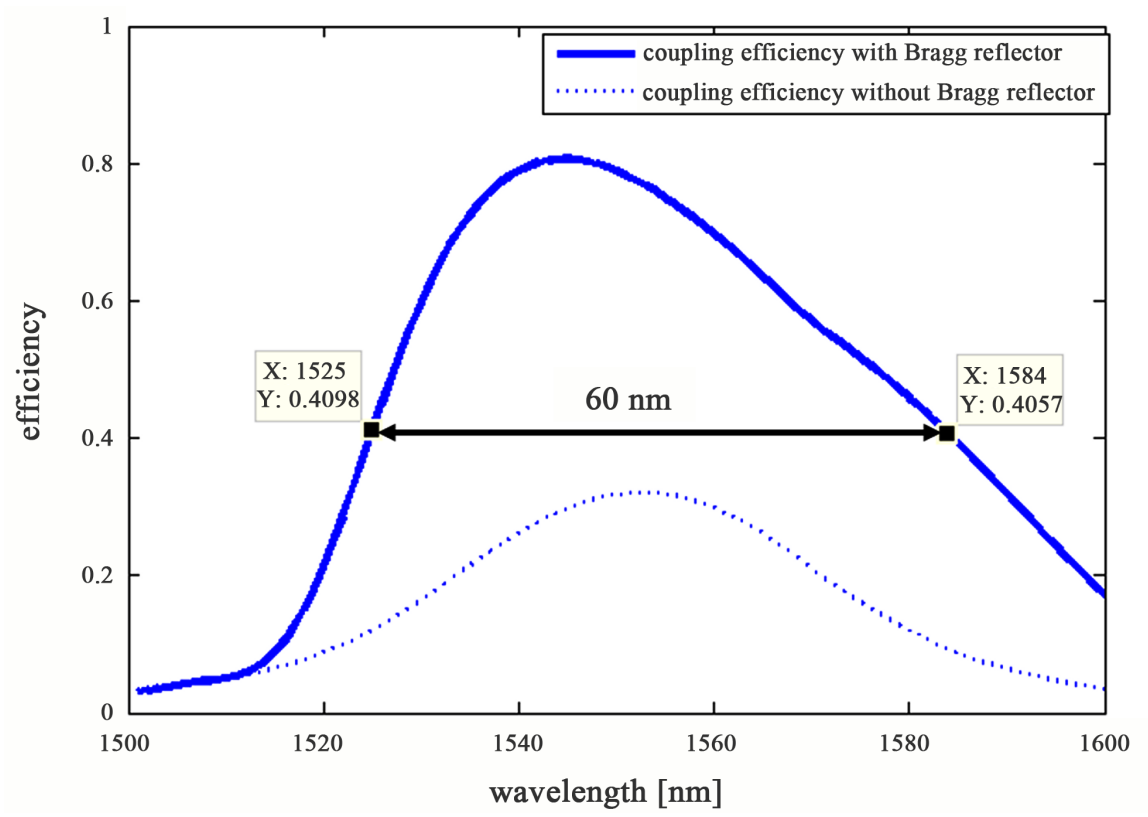

Figure 5. Grating coupling efficiency with reflector and without reflector.

Table 2. Design parameters of the improved $\mathrm{As}_{2} \mathrm{~S}_{3}$ grating coupler.

\begin{tabular}{cc}
\hline Parameter & Value \\
\hline $\mathrm{As}_{2} \mathrm{~S}_{3}$ thickness in Bragg reflector & $130 \mathrm{~nm}$ \\
$\mathrm{SiO}_{2}$ thickness in Bragg reflector & $340 \mathrm{~nm}$ \\
BCB thickness & $2 \mu \mathrm{m}$ \\
$\mathrm{As}_{2} \mathrm{~S}_{3}$ thickness for grating layer & $500 \mathrm{~nm}, 100 \mathrm{~nm}$ \\
$\mathrm{Thin}_{\text {film X-cut LiNbO }}$ thickness & $500 \mathrm{~nm}$ \\
Grating coupler period & $850 \mathrm{~nm}$ \\
Grating coupler duty cycle & $0.94,0.82,0.70,0.59$ \\
Number of periods & $2,2,2,16$ \\
\hline
\end{tabular}

a. For the first two periods, $\mathrm{DC}=0.94$; for the next two periods, $\mathrm{DC}=0.82$; then the next two $\mathrm{DC}=0.70$; for the rest 16 periods, $\mathrm{DC}=0.59$. 
from the efficiency peak. Spectrum symmetry can be further optimized by tweaking grating period.

\section{Fabrication}

A thin film X-cut $\mathrm{LiNbO}_{3}$ sample (from NanoLN) is cleaned using a Q-tip with acetone, methanol and isopropyl alcohol (IPA). This sample has $500 \mathrm{~nm} \mathrm{X-cut}$ thin film $\mathrm{LiNbO}_{3}$ on top of $2 \mu \mathrm{m} \mathrm{SiO}_{2}$ on Si substrate. We sputter $400 \mathrm{~nm} \mathrm{As} \mathrm{S}_{3}$, $130 \mathrm{~nm} \mathrm{SiO}$ and $40 \mathrm{~nm}$ Ti onto the sample. Then electron beam lithography (EBL) is performed for pattern definition. 2.25\% PMMA solution is used as positive EBL resist. The exposure dosage is $350 \mu \mathrm{C} / \mathrm{cm}^{2}$. After EBL, the sample is developed in fresh developer that is made from $40 \mathrm{~mL}$ IPA and $20 \mathrm{~mL}$ DI water. $\mathrm{O}_{2}$ Plasma ashing is then carried out to remove the residue PMMA resist at corner areas. $30 \mathrm{~nm}$ of $\mathrm{Ti}$ is deposited through e-beam evaporation, followed by a lift-off step in acetone.

Reactive ion etching (RIE) is performed to remove the metal layer and $\mathrm{As}_{2} \mathrm{~S}_{3}$ outside pattern areas. In order to make sure $\mathrm{As}_{2} \mathrm{~S}_{3}$ patterns are preserved, the sample needs to be taken out and checked under a microscope several times throughout RIE. After RIE, $100 \mathrm{~nm} \mathrm{SiO}_{2}$ is sputtered as a protective layer. A complete fabrication flow chart is shown in Figure 6.

In comparison, the improved structure is much more complicated. Figure 7 shows a tentative fabrication flow chart. Here, the top part of grating coupler starts with $\mathrm{As}_{2} \mathrm{~S}_{3}$ deposition (along with $\mathrm{SiO}_{2}$ and Ti protective layer deposition) on $\mathrm{LiNbO}_{3}$ thin film substrate. Then EBL is used to define grating coupler pattern. During RIE step, the etching time should be controlled so that the shallow layer has $400 \mathrm{~nm} \mathrm{As}_{2} \mathrm{~S}_{3}$ removed, while the pattern area preserve the $500 \mathrm{~nm}$ $\mathrm{As}_{2} \mathrm{~S}_{3}$. The bottom part starts with deposition of $\mathrm{As}_{2} \mathrm{~S}_{3}$ and $\mathrm{SiO}_{2}$ thin films as the Bragg reflector, following Table 2. Then the BCB solution is spin-coated and curing temperature $250^{\circ} \mathrm{C}$ for 60 minutes. Then more $\mathrm{BCB}$ solution is spin-coated, before the top part covers the solution, with $\mathrm{As}_{2} \mathrm{~S}_{3}$ in contact with $\mathrm{BCB}$. Then the whole device is cured at the same environment, for it to be well bonded. The last step is to remove the Si substrate for optical testing.

Table 3 summarizes fabrication complexity for both grating coupler structures. The improved grating coupler design has higher coupling efficiency, at the cost of additional deposition layers, more complicated etching, a substrate removal step and a bonding step.

\section{Results and Discussions}

After fabrication, the grating coupler is tested with a vertical fiber coupling setup. A source and a detector are integrated in a LUNA optical vector analyzer (OVA). The LUNA not only separates two polarization directions (TE and TM), but also provides time domain impulse response.

Figure 8 shows the insertion loss of a $2 \mathrm{~mm}$ long, $1.0 \mu \mathrm{m}$ wide $\mathrm{As}_{2} \mathrm{~S}_{3}$ waveguide with the basic grating couplers on both ends. Between each grating 


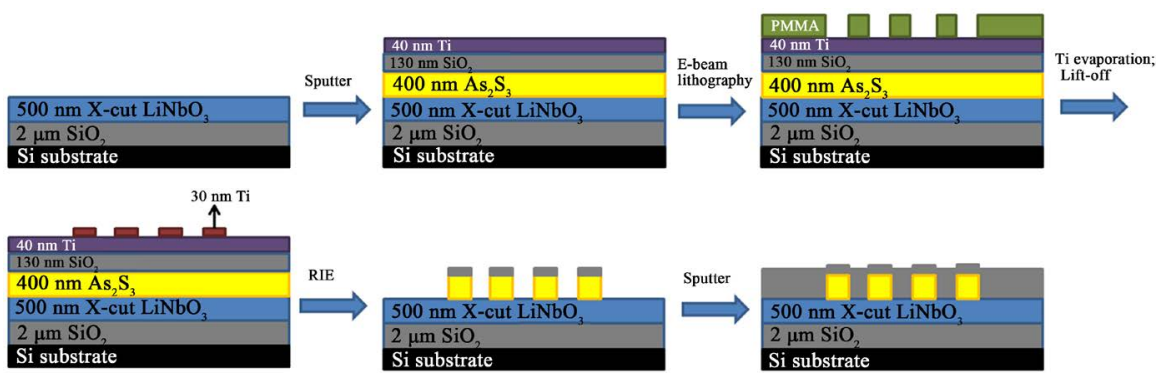

Figure 6. Fabrication of the basic grating coupler structure (side view).

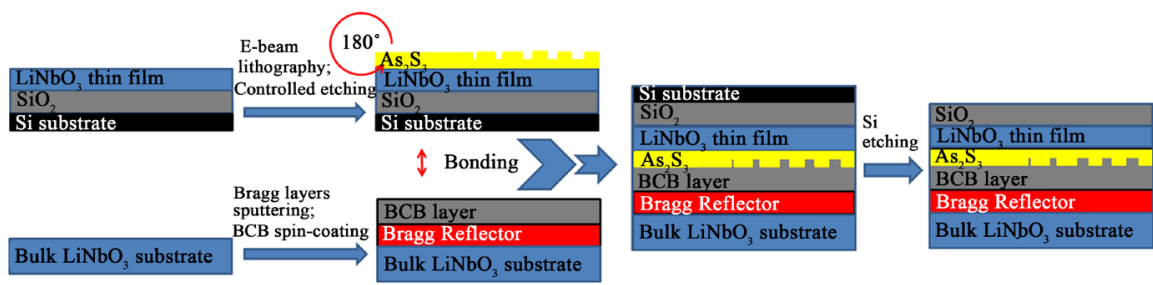

Figure 7. Proposed fabrication flow of the improved structure (side view).

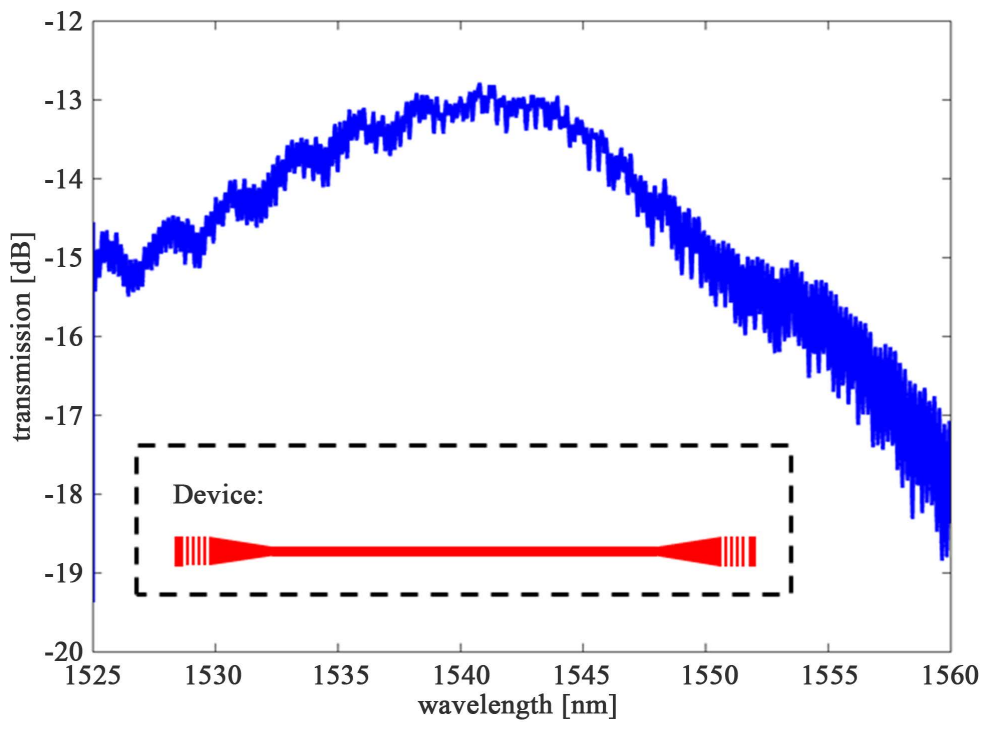

Figure 8. Insertion loss of the basic grating couplers with an $\mathrm{As}_{2} \mathrm{~S}_{3}$ waveguide.

Table 3. Comparison between two grating couplers.

\begin{tabular}{ccc}
\hline Parameter & Basic structure & Improved structure \\
\hline Deposition layers & 4 & 10 \\
Requires BCB bonding & No & Yes \\
Requires controlled etching & No & Yes \\
Requires substrate removal & No & Yes \\
Coupling efficiency & $53.0 \%$ & $78.8 \%$ \\
\hline
\end{tabular}

coupler and the waveguide, there is a $200 \mu \mathrm{m}$ long linear taper, with the wider tip of $12 \mu \mathrm{m}$ connecting the grating coupler and the narrower tip of $1 \mu \mathrm{m}$ con- 
necting the waveguide. The grating coupler period is $0.85 \mu \mathrm{m}$. Due to the fabrication imperfection, the peak transmission wavelength is shifted to the shorter wavelength range. As a rough estimation, the propagation loss at $1540 \mathrm{~nm}$ is 2.0 $\mathrm{dB} / \mathrm{cm}$. Assuming that the mode conversion loss in both tapers is negligible and the waveguide loss is wavelength-independent, the lowest fabricated grating coupler loss is $6.3 \mathrm{~dB}$ at $1540 \mathrm{~nm}$. Compared to the experimental result in [11], the grating coupler efficiency in this work is $2.55 \mathrm{~dB}$ worse, due to the fact that there is no Bragg reflector in this basic grating coupler structure, and the light is partially leaked through substrate.

The difference between measured grating coupler loss and simulation result is due to several factors: first, the overall insertion loss includes the SMF tails that are used to couple the OVA source/detector to the grating couplers. Since it is not convenient to isolate the fiber tails from the device under test (DUT), the surface qualities of those fiber tails and light propagation loss inside them would result in higher calculated grating coupler loss. Similarly, the tapers' loss might also be one contributing factor to the overall loss. In terms of fabrication, surface roughness caused by topology of the grating coupler can also result in lower coupling efficiency than theoretical prediction. During measurement, any small angular offset of the sample orientation would cause additional loss.

In order to improve the grating coupler performance, the grating period and DC need to be tweaked from the design values based on the current experimental results, since the actually materials and their refractive indices are off from the material database to some extent. Longer taper could be included in order to further reduce mode-conversion loss.

The basic grating coupler can be used to measure $\mathrm{As}_{2} \mathrm{~S}_{3}$ grating cavity structure on thin film $\mathrm{LiNbO}_{3}$. With electrode patterned on both sides of the cavity waveguide, it becomes EO tunable filters. Grating cavity reflection response can be extracted by truncation of the time domain impulse reflection (including grating coupler), followed by inverse Fourier transformation. Figure 9(a) shows the whole device, highlighting the grating cavity section in dashed box. Figure 9(b) shows the reflected spectrum of one fabricated Fabry-Perot (FP) cavity extracted in this manner. This device is constructed by 1000 periods of sidewall Bragg gratings, with period of $360 \mathrm{~nm}$ and grating depth of $200 \mathrm{~nm}$. The cavity length is $3 \mathrm{~mm}$ and the cavity width is $1 \mu \mathrm{m}$. The fitted result in Figure $9(\mathrm{c})$ is calculated by implementing coupled-mode theory (CMT) and transfer matrix method (TMM) with the fitting parameters listed in Table 4.

Optical confinement factor in $\mathrm{LiNbO}_{3}$ layer is a key parameter for EO tuning applications. Higher confinement factor in $\mathrm{LiNbO}_{3}$ layer means more effective EO tuning, since $\mathrm{LiNbO}_{3}$ crystal is the only EO tunable material in this structure. By decreasing $\mathrm{As}_{2} \mathrm{~S}_{3}$ waveguide width, confinement factor in thin film $\mathrm{LiNbO}_{3}$ can be effectively increased, as shown in Figure 10. $500 \mathrm{~nm} \mathrm{X-cut} \mathrm{LiNbO}_{3}$ on top of $2 \mu \mathrm{m} \mathrm{SiO}_{2}$ on a $\mathrm{Si}$ wafer is used in the simulation. With $0.4 \mu \mathrm{m}$ waveguide width, the confinement factor is $82.3 \%$. 


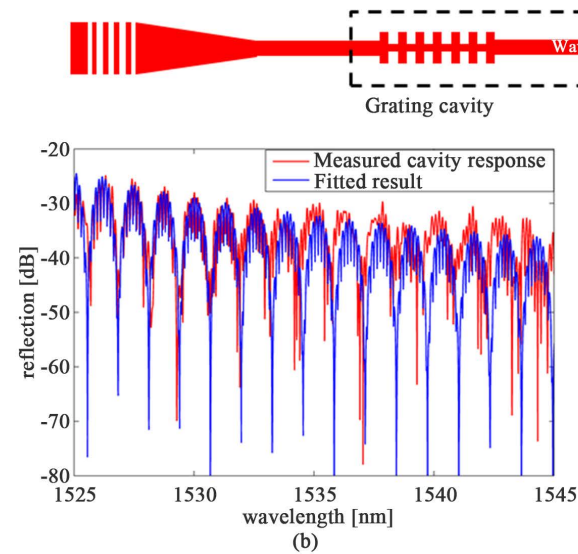

(a)

Figure 9. (a) Grating cavity device under test. The dashed box is the grating cavity section; (b) Reflection spectrum of a fabricated grating cavity device; (c) Zoom-in reflection.

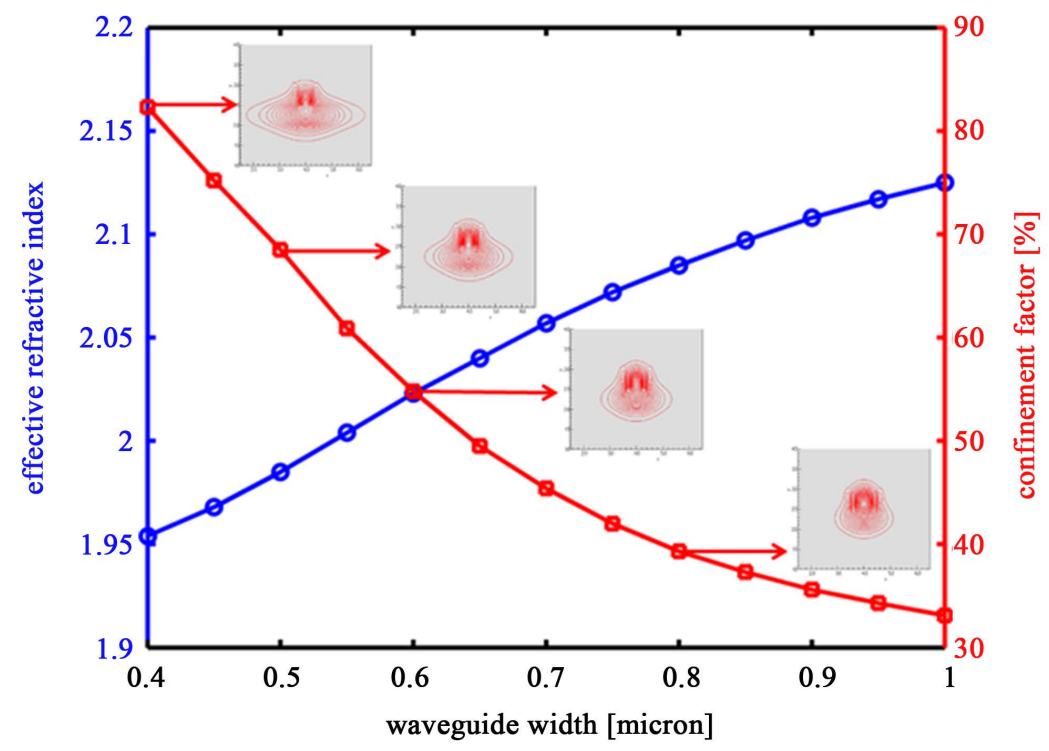

Figure 10. Effective refractive index of the hybrid and optical confinement factor in thin film $\mathrm{LiNbO}_{3}$ change with different $\mathrm{As}_{2} \mathrm{~S}_{3}$ waveguide widths.

Table 4. Fitting parameters for the grating cavity reflection response.

\begin{tabular}{cc}
\hline Fitting parameter & Value \\
\hline Coupling coefficient & $0.85 \mathrm{~mm}^{-1}$ \\
\hline Effective refractive index & 2.11 \\
\hline Group refractive index & 2.6 \\
\hline Waveguide loss & $2.0 \mathrm{~dB} / \mathrm{cm}$ \\
\hline
\end{tabular}

\section{Conclusions}

$\mathrm{As}_{2} \mathrm{~S}_{3}$ grating coupler on thin film $\mathrm{LiNbO}_{3}$ is designed in order to open the door to multiple EO applications with thin film $\mathrm{LiNbO}_{3}$. A basic grating coupler structure is fabricated and the experimental results show $23.4 \%$ coupling efficiency while the simulation is at $53.0 \%$. The efficiency difference is due to fabri- 
cation imperfection, testing fibers, and misalignment during measurement. An improved grating coupler is designed, and the theoretical coupling efficiency can be as high as $78.8 \%$ at $1550 \mathrm{~nm}$ with $60 \mathrm{~nm}$ bandwidth.

$\mathrm{As}_{2} \mathrm{~S}_{3}$ waveguide propagation loss of $2.0 \mathrm{~dB} / \mathrm{cm}$ is calculated through TMM fitting a grating cavity measurement result enabled by the $\mathrm{As}_{2} \mathrm{~S}_{3}$ grating coupler. A simulation of $\mathrm{As}_{2} \mathrm{~S}_{3}$ waveguide cross-section on a $500 \mathrm{~nm}$ thin film $\mathrm{LiNbO}_{3}$ result indicates as high as $82.3 \%$ optical confinement in $\mathrm{LiNbO}_{3}$ layer if the $\mathrm{As}_{2} \mathrm{~S}_{3}$ cavity waveguide width is reduced to $0.4 \mu \mathrm{m}$. This means that high EO tuning efficiency can be achieved on thin film $\mathrm{LiNbO}_{3}$ layer, once an electric field is properly applied across the cavity waveguide section.

\section{Acknowledgements}

The authors would thank Dr. Long Chang from The University of Houston Nanofabrication Facility for valuable discussion on E-beam lithography.

\section{References}

[1] Snider, W.T., Macik, D.D. and Madsen, C.K. (2012) Electro-optically Tunable Mach-Zehnder Interferometer on LiNbO3 Substrate. IEEE Photonics Technology Letters, 24, 1415-1417. https://doi.org/10.1109/LPT.2012.2205377

[2] Macik, D.D. and Madsen, C.K. (2016) Fabrication of $\mathrm{LiNbO}_{3}-\mathrm{As}_{2} \mathrm{~S}_{3}$ Waveguides for Beam Steering Applications. Proceedings of SPIE, San Diego, 14 September 2016, Article ID: 99700H-10.

[3] Wooten, E.L., Kissa, K.M., Yi-Yan, A., Murphy, E.J., Lafaw, D.A., Hallemeier, P.F., Maack, D., Attanasio, D.V., Fritz, D.J., McBrien, G.J. and Bossi, D.E. (2000) A Review of lithium Niobate Modulators for Fiber-Optic Communications Systems. IEEE Journal of Selected Topics in Quantum Electronics, 6, 69-82. https://doi.org/10.1109/2944.826874

[4] Rao, A., et al. (2015) Heterogeneous Microring and Mach-Zehnder Modulators Based on Lithium Niobate and Chalcogenide Glasses on Silicon. Optics Express, 23, 22746-22752. https://doi.org/10.1364/OE.23.022746

[5] Guarino, A., Poberaj, G., Rezzonico, D., Degl'Innocenti, R. and Günter, P. (2007) Electro-Optically Tunable Microring Resonators in Lithium Niobate. Nature Photonics, 1, 407-410. https://doi.org/10.1038/nphoton.2007.93

[6] Levy, M., Osgood, R., Liu, R., Cross, L., Cargill, G., Kumar, A., et al. (1998) Fabrication of Single-Crystal Lithium Niobate Films by Crystal Ion Slicing. Applied Physics Letters, 73.

[7] Ramadan, T.A., Levy, M. and Osgood, R. (2000) Electro-Optic Modulation in Crystal-Ion-Sliced z-Cut LiNbO3 Thin Films. Applied Physics Letters, 76, 1407. https://doi.org/10.1063/1.126046

[8] Lu, H., Sadani, B., Courjal, N., Ulliac, G., Smith, N., Stenger, V., et al. (2012) Enhanced Electro-Optical Lithium Niobate Photonic Crystal Wire Waveguide on a Smart-Cut Thin Film. Optics Express, 20, 2974-2981.

https://doi.org/10.1364/OE.20.002974

[9] Wang, X., Zhou, Y. and Madsen, C.K. (2014) Fabrication and Measurement of Sidewall Gratings Integrated in Hybrid As2S3-Ti:LiNbO3 Optical Waveguides. Journal of Lightwave Technology, 32, 2989-2995.

https://doi.org/10.1109/JLT.2014.2334054 
[10] Zhou, Y., Wang, X., Zhang, C. and Madsen, C.K. (2015) Resonant Enhanced Low-Power Nonlinear Tuning Capability Using an $\mathrm{As}_{2} \mathrm{~S}_{3}$ Waveguide on $\mathrm{LiNbO}_{3}$. Proceedings of SPIE, San Francisco, 2 April 2015, 936514.

[11] Zhang, C. and Madsen, C.K. (2016) Demonstration of an As2S3-on-LiNbO3 Grating Coupler and Its Application of Measuring a Grating-Based Filter. IEEE Photonics Journal, 8, 1-7. https://doi.org/10.1109/JPHOT.2016.2616220

[12] Polyanskiy, M.N. (2017) Refractive Index Database. https://refractiveindex.info

[13] Dakss, M.L., Kuhn, L., Heidrich, P.F. and Scott, B.A. (1970) Grating Coupler for Efficient Excitation of Optical Guided Waves in Thin Films. Applied Physics Letters, 16, 523-525. https://doi.org/10.1063/1.1653091

[14] Niklaus, F., Enoksson, P., Kälvesten, E. and Stemme, G. (2001) Low-Temperature Full Wafer Adhesive Bonding. Journal of Micromechanics and Microengineering, 11, 100. https://doi.org/10.1088/0960-1317/11/2/303

[15] Niklaus, F., Andersson, H., Enoksson, P. and Stemme, G. (2001) Low Temperature Full Wafer Adhesive Bonding of Structured Wafers. Sensors and Actuators A: Physical, 92, 235-241. https://doi.org/10.1016/S0924-4247(01)00568-4

[16] Processing Procedures for BCB Adhesion. DOW. http://www.dow.com/cyclotene/docs/bcb_adhesion.pdf 